\title{
Investigating the Impacts of Longitudinal and Lateral Distances on the Lift and Drag Coefficients of two Closely Moving Vehicles
}

\author{
Mohammadreza Saber Ashkezari ${ }^{1}$, Ali Mohammad ${ }^{1}$, Masoud Darbandi ${ }^{1}$, Gerry E. Schneider ${ }^{2}$ \\ ${ }^{1}$ Department of Aerospace Engineering, Center of Excellence in Aerospace Systems, Sharif University of Technology \\ Tehran, P. O. Box 11365-11155, Postcode 14588-89694, Iran \\ mohammadreza.saber@ae.sharif.edu; Mohammad.ali@ae.sharif.edu; darbandi@sharif.edu \\ ${ }^{2}$ Department of Mechanical and Mechatronics Engineering, University of Waterloo \\ Waterloo, Ontario, N2L 3G1, Canada \\ gerry.schneider@uwaterloo.ca
}

\begin{abstract}
The limitations in using conventional wind tunnels and rapid developments in computer hardware have led to considerable efforts to study the vehicle aerodynamics using the computational fluid dynamic (CFD) capabilities for the last decade. The main objective of this paper is to investigate the changes in lift and drag coefficient of two closely moving vehicles subject to their lateral and longitudinal distances. We investigate two longitudinal distances of 0.2 and $2 \mathrm{~m}$ and two lateral distances of 0.2 and $1 \mathrm{~m}$ in this study. Simplified vehicle geometry, say the standard Ahmed body model, is used as the benchmark vehicle to carry on the investigation. The CFD methods are used to compute the flow patterns around the vehicle. The investigation in longitudinal distance shows that the drag coefficient of both vehicles significantly decreases, specifically the front one. Also, the lift coefficients of both vehicles reduce, and this force transforms to downforce for the rear vehicle. The investigation in lateral distance indicates that the drag coefficient depends on the attributed distance; however, the lift coefficient reduces in both distances. In the lateral distance, one expects equal coefficients for both vehicles; however, the results show that there is slight difference between them.
\end{abstract}

Keywords: CFD, Ahmed body, Drag Coefficient, Lift Coefficient, Lateral Distance, Longitudinal Distance.

\section{Introduction}

Optimized exposed-car-body aerodynamics design is necessary to achieve efficient vehicles to decrease fuel consumption, $\mathrm{CO} 2$ emissions, and noise generation while keeping good driving stability. Recent advances in computer calculation have created new opportunities for researchers to investigate these vital topics in the automobile industry. The promotion has been raising the importance of simulating the external aerodynamics of vehicles and computing the corresponding aerodynamic characteristic numbers, and exposed body optimization of vehicles will be obtained during this analysis. Despite an efficient design, we aim to investigate how much change in situations affects the cars' lift and drag coefficients.

Ahmed et al. [1] analysed the time-averaged wake structure around the Ahmed body, a primary ground vehicle type of bluff body, at a Reynolds number equal to $1.2 \mathrm{e} 6$ by manipulating the rear slant angle the range of $0-40^{\circ}$ in increments of $5^{\circ}$. However, the study did not yield information about unsteady flow characteristics of the flow around the Ahmed body. Meile and Brenn [2] investigated the aerodynamic behaviour of the Ahmed body experimentally and numerically. The experiments cover the two slant angles, $25^{\circ}$ and $35^{\circ}$, and Reynolds numbers up to $2.784 \mathrm{e} 6$. Kumar and Narayan [3] aimed to reduce the drag force by examining the influence of different types of spoilers on a sedan c, leading to the decline of $2.18 \%$ in the drag coefficient. Blocken and Toparlar [4] presented the results of CFD simulations and wind tunnel measurements to ascertain and quantify the upstream effect of the mentioned car on the cyclist's drag in front of it. They investigated the drag reduction for the cyclist ranges from $3.7 \%$ over $1.4 \%$ to $0.2 \%$ for realistic separation distances of 3,5 , and $10 \mathrm{~m}$, respectively.

Minguez, et al. [5] evaluated the turbulent flow structure over a simplified automotive model, the Ahmed body with a $25^{\circ}$ slanted back face, using high-order large-eddy simulations (LESs) at Reynolds number of 7.68e5. Banga and Zunaid [6] analysed a solution to reduce the inefficiencies and losses caused due to significant parameters on the aerodynamics of 
road vehicles (drag, lift). They accomplished this by examining different rear slant angles of the Ahmed body and its effect on the drag and lift coefficients and determined the optimum angle for least drag through numerical simulation. Mohammadikalakoo, et al. [7] concentrated on the interaction of a car body with the surrounding airflow to provide small changes for reducing vehicle fuel consumption by reducing drag. There are many passive and active methods for this purpose. The main goal was to develop passive drag reduction devices for the simplified reference model (Ahmed body with $25^{\circ}, 30^{\circ}$, and $35^{\circ}$ slant angle) used to represent bluff body vehicles. Passive flow control was performed by adding linking tunnels at the rear of the bluff body, blowing the flow from the sidewalls (high-pressure zone) to the wake region (low-pressure zone). The movement of a high-pressure flow to the wake zone reduces the wake size, which decreases the pressure drag. The optimal configuration was reached by numerical analysis (showing a drag reduction of up to 5\%); meanwhile, experimental tests were performed in a wind tunnel to check the effect of this passive flow control on an Ahmed body $25^{\circ}$. Siddiqui and Agelin-Chaab [8] reported a numerical investigation of aspect ratio (AR) effect on the flow structure around the $35^{\circ}$ Ahmed body. The AR was defined as the ratio of length to the height of the model. A total of five ARs were considered at a Reynolds number of 7.8e 5 based on the height of Ahmed body. Buscarioloa, et al. [9] extended the geometrical variation to evaluate the influence of a rear underbody diffuser commonly applied in high-performance and race cars to improve downforce. Parametric studies were performed on the rear diffuser angle of two baseline configurations of the Ahmed body: the first with a $0^{\circ}$ upper slant angle and the second with a $25^{\circ}$ slant angle.

This paper considers different lateral and longitudinal distances of two Ahmed bodies moving closely on their lift and drag coefficients. In most of the previous articles, the authors have sought simplifications to facilitate car aerodynamic coefficient calculations. This paper aims to elucidate how much these coefficients will change the effect of another vehicle.

\section{Governing Equation}

The continuity and momentum equations (Navier-Stokes equations) applied to a fluid passing through an infinitesimal; fixed control volume yields the following equations:

$$
\begin{gathered}
\frac{\partial \rho}{\partial t}+\nabla \cdot(\rho V)=0 \\
\rho \frac{\partial V}{\partial t}+\rho(V . \nabla) V=-\nabla p+\mu \nabla^{2} V+\beta
\end{gathered}
$$

where $\rho$ is density, $t$ is time, $\nabla$ is the vector differential operator, $V$ is the velocity vector, $p$ is static pressure, $\mu$ is fluid viscosity, and $\beta$ is body force [10].

For the problem analysed in this paper, the realizable $\mathrm{k}-\varepsilon$ turbulent model is used. The $k-\varepsilon$ model is one of the most common turbulent models. It is a semi-empirical, two-equation model, which means it includes two transport equations to represent the turbulent properties of flow. The first transport variable is the turbulent kinetic energy $k$. The second transport variable is the turbulent dissipation $\varepsilon$. It is the variable that determines the scale of the turbulence, whereas the first variable, $k$, determines the energy in the turbulence. They are defined as

$$
\begin{gathered}
\frac{\partial}{\partial t}(\rho k)+\frac{\partial}{\partial x_{j}}\left(\rho k u_{j}\right)=\frac{\partial}{\partial x_{j}}\left[\left(\mu+\frac{\mu_{t}}{\sigma_{k}}\right) \frac{\partial k}{\partial x_{j}}\right]+G_{k}+G_{b}-\rho \varepsilon-Y_{M}+S_{k} \\
\frac{\partial}{\partial t}(\rho \varepsilon)+\frac{\partial}{\partial x_{j}}\left(\rho \varepsilon u_{j}\right)=\frac{\partial}{\partial x_{j}}\left[\left(\mu+\frac{\mu_{t}}{\sigma_{\varepsilon}}\right) \frac{\partial \varepsilon}{\partial x_{j}}\right]+\rho C_{1} S_{\varepsilon}-\rho C_{2} \frac{\varepsilon^{2}}{k+\sqrt{v \varepsilon}}+C_{1 \varepsilon} \frac{\varepsilon}{k} C_{3 \varepsilon} G_{b}+S_{\varepsilon} \\
C_{1}=\max \left[0 \cdot 43 \cdot \frac{\eta}{\eta+5}\right] \cdot \eta=S \frac{k}{\varepsilon} \cdot S=\sqrt{2 S_{i j} S_{i j}}
\end{gathered}
$$

In these equations, $G_{k}$ represents the generation of turbulence kinetic energy due to the mean velocity gradients, $G_{b}$ is the generation of turbulence kinetic energy due to buoyancy, $Y_{M}$ represents the contribution of the fluctuating dilatation incompressible turbulence to the overall dissipation rate. $C_{2}$ and $C_{1 \varepsilon}$ are constant. $\sigma_{k}$ and $\sigma_{\varepsilon}$ are the turbulent Prandtl 
numbers for $k$ and $\varepsilon$, respectively. $S_{k}$ and $S_{\varepsilon}$ are user-defined source terms. The model constants $C_{2}, \sigma_{k}$, and $\sigma_{\varepsilon}$ have been established to ensure that the model performs well for specific canonical flows. The model constants are $C_{1 \varepsilon}=1$. 44. $\mathrm{C}_{2}=1 \cdot 9$. $\sigma_{\mathrm{k}}=1 \cdot 0$ and $\sigma_{\varepsilon}=1 \cdot 2[11]$.

\section{Geometry, Grid Generation, And Numerical Validation}

The present study has been validated by comparing the corresponding results of Meile and Brenn [2]. The slant angle of $35^{\circ}$ and Reynolds numbers of $2.784 \mathrm{e} 6$ (velocity of $40 \mathrm{~m} / \mathrm{s}$ ) is investigated. The geometrical shape of the Ahmed body is depicted in Fig. 1. Despite considerable deviation of its geometry from the usual vehicles, the body represents the basic aerodynamic properties, especially in the rear part. The slant angle $\phi$ has a strong influence on the aerodynamic drag and lift at the back. The body was first proposed by Ahmed et al. [1]. The reference Ahmed body is $1044 \mathrm{~mm}$ long, $389 \mathrm{~mm}$ wide, and $288 \mathrm{~mm}$ height. To reduce computational cost, only half of Ahmed body is generated. Basically, proper boundary conditions are dedicated to the duplicated boundaries. A single body domain of air is created surrounding the Ahmed body walls after subtracting it from the air enclosure. It has dimensions of $5 \mathrm{~m}$ from the front, $7.5 \mathrm{~m}$ from the rear, $3 \mathrm{~m}$ from the top, and $2 \mathrm{~m}$ in width. The model is $50 \mathrm{~mm}$ above the floor.

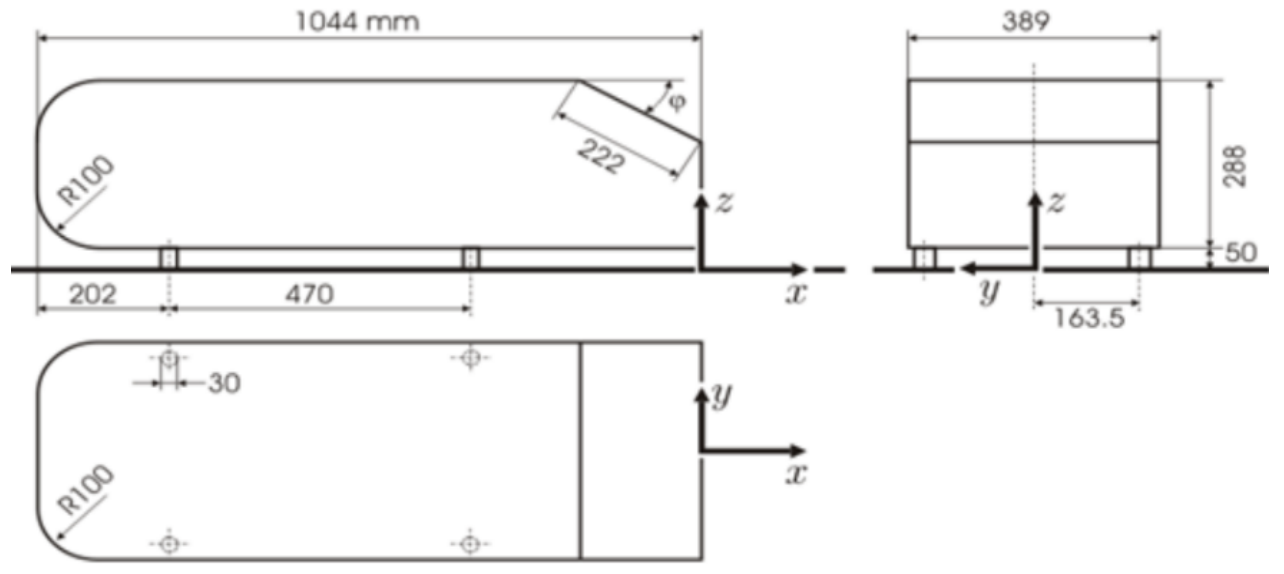

Fig. 1: Ahmed body geometrical dimensions [1]

Different sizes are used in order to reach an accurate and efficient grid. The domain has separated into two regions to creating smaller grids near Ahmed body. In the validation, 1,888,696 grids are produced, and this value differs in the lateral and longitudinal investigation. The settings are shown in Table 1, and the generated grids are depicted in Fig. 2.

Table 1: Grid specification

\begin{tabular}{|l|c|}
\hline \multicolumn{1}{|c|}{ Settings } & Value \\
\hline Max. size of elements in domain & $250 \mathrm{~mm}$ \\
\hline Max. size of mesh in wake domain & $15 \mathrm{~mm}$ \\
\hline Max. size of mesh around \& under the body & $8 \mathrm{~mm}$ \\
\hline Size of mesh around body's legs & $3 \mathrm{~mm}$ \\
\hline Inflation layer method & First aspect ratio of 5, growth rate of 20\% \& 5 layers \\
\hline Total number of elements & $1,888,696$ \\
\hline
\end{tabular}




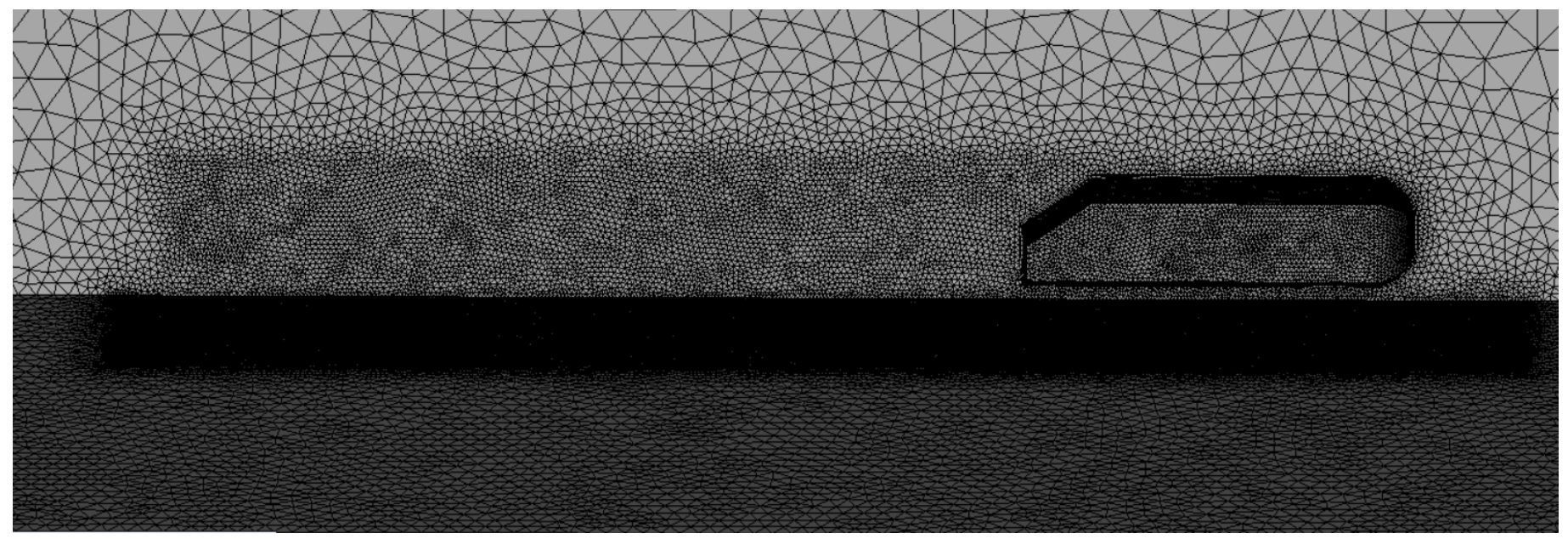

Fig. 2: Generated grids

The realizable $k$-epsilon model with enhanced wall function for near-wall treatment is used with Inlet velocity of 40 $\mathrm{m} / \mathrm{s}$ and turbulence intensity is about $1 \%$, and turbulence viscosity of 10 at the inlet; turbulence intensity near 5\% and turbulence viscosity ratio of 10 is considered at the outlet surface. Incompressible dry air flows in the domain as the fluid part with a temperature of $288.16 \mathrm{~K}$, and viscosity of $1.7894 \mathrm{e}-05 \mathrm{~kg} / \mathrm{m}$.s. Uniform velocity normal to the boundary is assigned at the inlet surface along with constant atmospheric pressure at the outlet boundary. Symmetry B.C. is allocated to the lateral, upper wall and the moving wall at the floor boundaries. Eventually, the solution method utilizes a pressurevelocity coupling scheme. Simulation results and corresponding data from the literature and experimental results from the wind tunnel are shown in Table 2 that will validate our simulation.

Table 2: Validation Results

\begin{tabular}{|c|c|c|c|}
\hline & Our Simulation & Literature Simulation & Experimental Results \\
\hline Drag Coefficient & 0.272 & 0.276 & 0.279 \\
\hline Lift Coefficient & 0.0143 & 0.013 & 0.004 \\
\hline
\end{tabular}

\section{The Results}

\subsection{The Longitudinal Distance Study}

This section examines the lift and drag coefficient for two different longitudinal distances of Ahmed bodies. The car near the inlet is designated as the "front car," and another car is the "rear car". The lift and drag coefficients are measured and shown in Table 3 for two different distances, 0.2 and $2 \mathrm{~m}$, respectively. By comparing single-car (validation) results and these results, it is concluded that the lift coefficient for both cars has decreased. Lift force for the rear car at $0.2 \mathrm{~m}$ transferred to downforce since the car experiences a high-pressure region over the body. Also, the front car at $2 \mathrm{~m}$ faces downforce for the low-pressure region beneath it. Therefore, the force generated on the higher surfaces is more significant than on the lower surfaces. Moreover, the drag coefficient of the rear car is almost the same, while the value for the front car cut down significantly in $0.2 \mathrm{~m}$ longitudinal distances. Since the front car has a smaller separation and pressure gradient region, less pressure drag is calculated. 
Table 3. Lift and drag coefficients in different longitudinal distances

\begin{tabular}{|c|c|c|c|c|}
\hline Distance (m) & \multicolumn{2}{|c|}{0.2} & \multicolumn{2}{c|}{2} \\
\hline Car & Rear Car & Front Car & Rear Car & Front Car \\
\hline Drag Coefficient & 0.256 & 0.103 & 0.273 & 0.250 \\
\hline Lift Coefficient & -0.00161 & 0.00569 & 0.0040 & -0.00021 \\
\hline
\end{tabular}

While the air flows over the vehicle's exposed surface, some points face stalls due to the change in velocity; hence the fluid starts flowing in the reverse direction. In other words, the separation of the fluid flow happens. It usually occurs at the rear part of the vehicles and it depends mainly on the pressure distribution imposed by the outer layer of the flow [11]. The separation causes the flow to change its behaviour behind the vehicle and thereby affects the flow field. This phenomenon is the primary factor to be considered while studying the wake of a car. Flow separation is unfavourable because it leads to a larger wake and less pressure on the rear surface, reducing pressure recovery.

On the other hand, the front part of the flow field confronts a high-pressure value area, whereas the rear parts separate, leading to a high suction in the car flow field. As the force component created by such a gradient of pressure changes, the result is called 'Pressure Drag.' This factor is affected by the height of the vehicle and the separation amount in the flow field. The flow separation is shown in velocity path lines figures, and also different pressure regions are pinpointed in the static pressure contour plots.

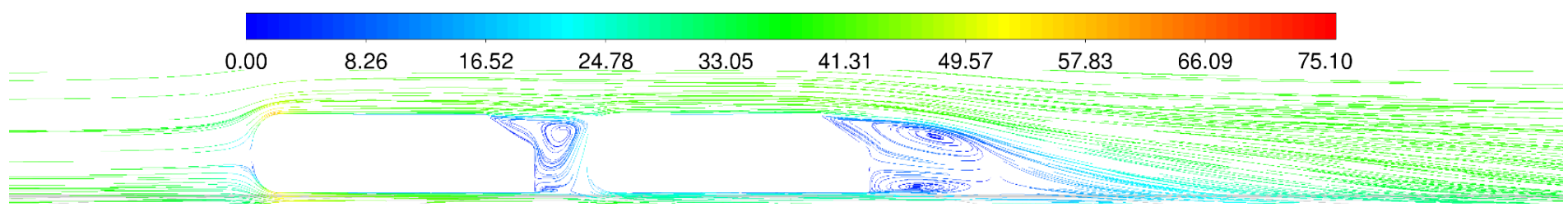

Fig. 3: Velocity path lines of $0.2 \mathrm{~m}$ longitudinal distance

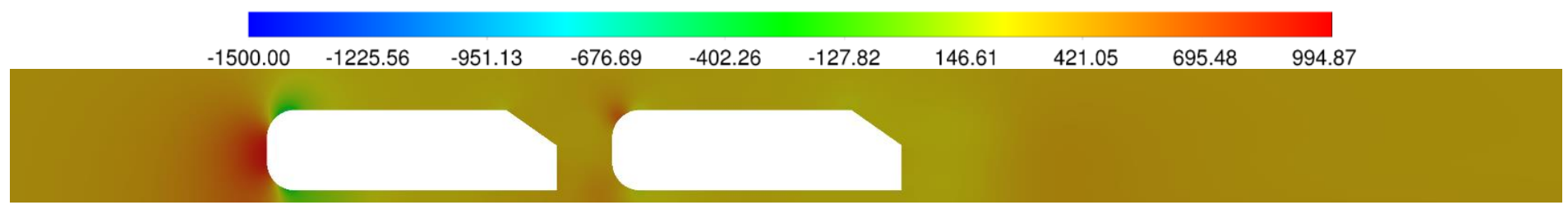

Fig. 4: Static pressure contour of $0.2 \mathrm{~m}$ longitudinal distance

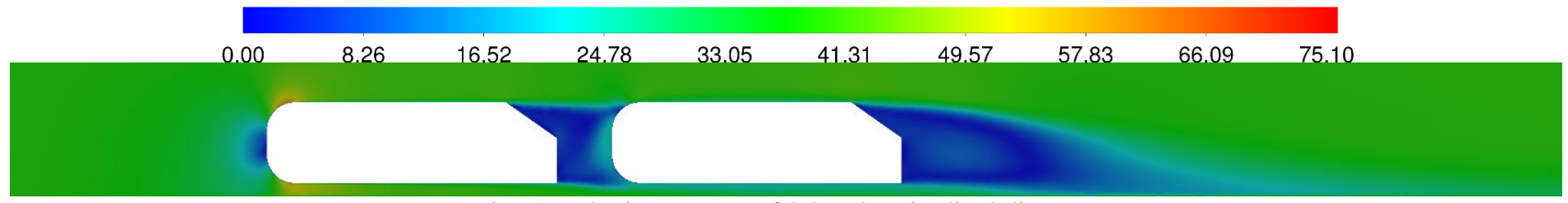

Fig. 5: Velocity contour of $0.2 \mathrm{~m}$ longitudinal distance

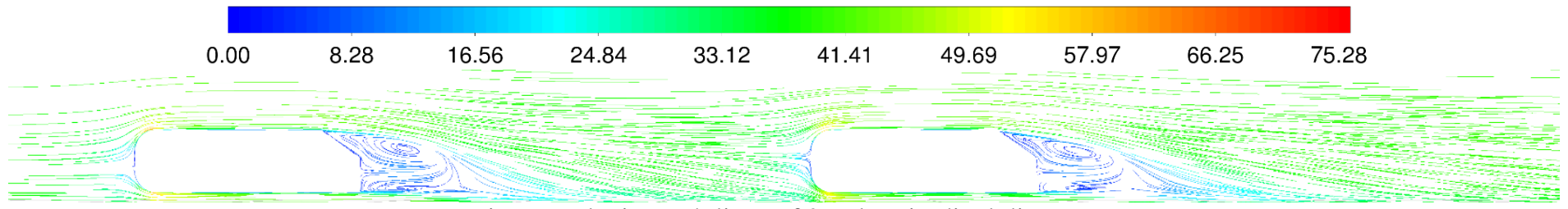

Fig. 6: Velocity path lines of $2 \mathrm{~m}$ longitudinal distance 


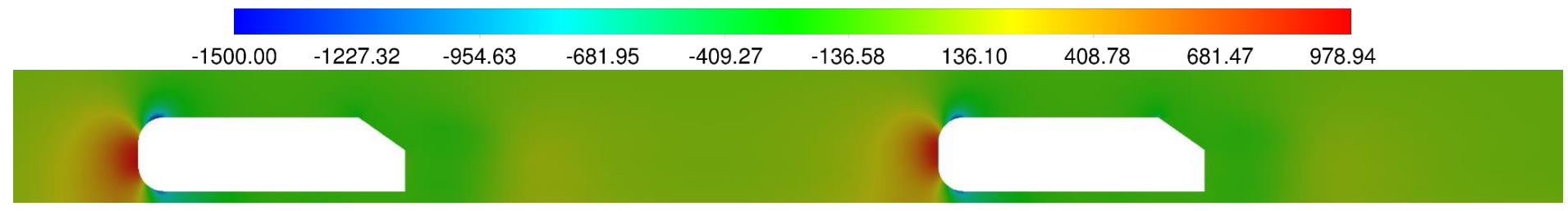

Fig. 7: Static pressure contour of $2 \mathrm{~m}$ longitudinal distance

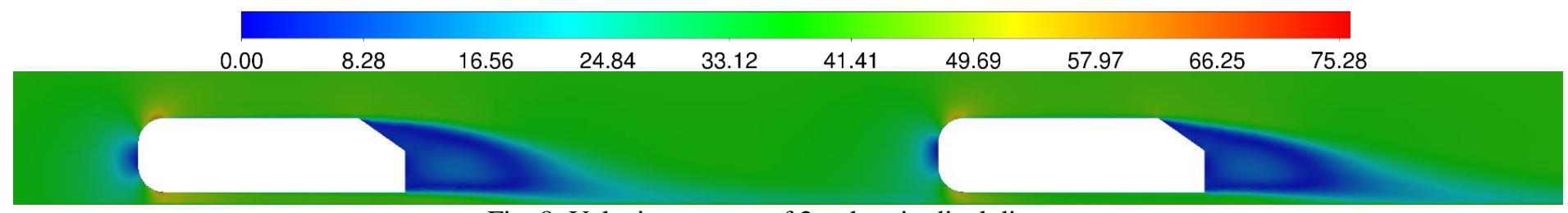

Fig. 8: Velocity contour of $2 \mathrm{~m}$ longitudinal distance

\subsection{The Lateral Distance Study}

This section investigates the lift and drag coefficient for two different lateral distances of Ahmed bodies. For designation, consider the flow entering the domain like a flow particle, and then define the cars as the left and the right car. It is necessary to mention that recognition of the half bodies became so complicated in this part. To solve this issue, two entire shape bodies, as left and right cars were generated. Two different lateral distances of $0.2 \mathrm{~m}$ and $1 \mathrm{~m}$ were investigated, respectively, with a fluid domain of 4 and $5 \mathrm{~m}$ lengths. The lift and drag coefficients are shown in Table 4 .

Table 4. Lift and drag coefficients of lateral distances

\begin{tabular}{|c|c|c|c|c|}
\hline Distance [m] & \multicolumn{2}{|c|}{0.2} & \multicolumn{2}{c|}{1} \\
\hline Car & Left Car & Right Car & Left Car & Right Car \\
\hline Drag Coefficient & 0.286 & 0.292 & 0.267 & 0.269 \\
\hline Lift Coefficient & -0.00591 & -0.00426 & -0.00216 & 0.00189 \\
\hline
\end{tabular}

Figure 8 plots the lift and drag coefficients for ten time steps of $2 \mathrm{~s}$ in the transient mode. More details are provided in Fig. 9 and Fig. 10. There is a slight distinction between the left and right cars. One probable reason might be the impact of turbulence flow or wake regions that bring irregularity in the flow field. Nevertheless, likewise trend is followed by both cars. 

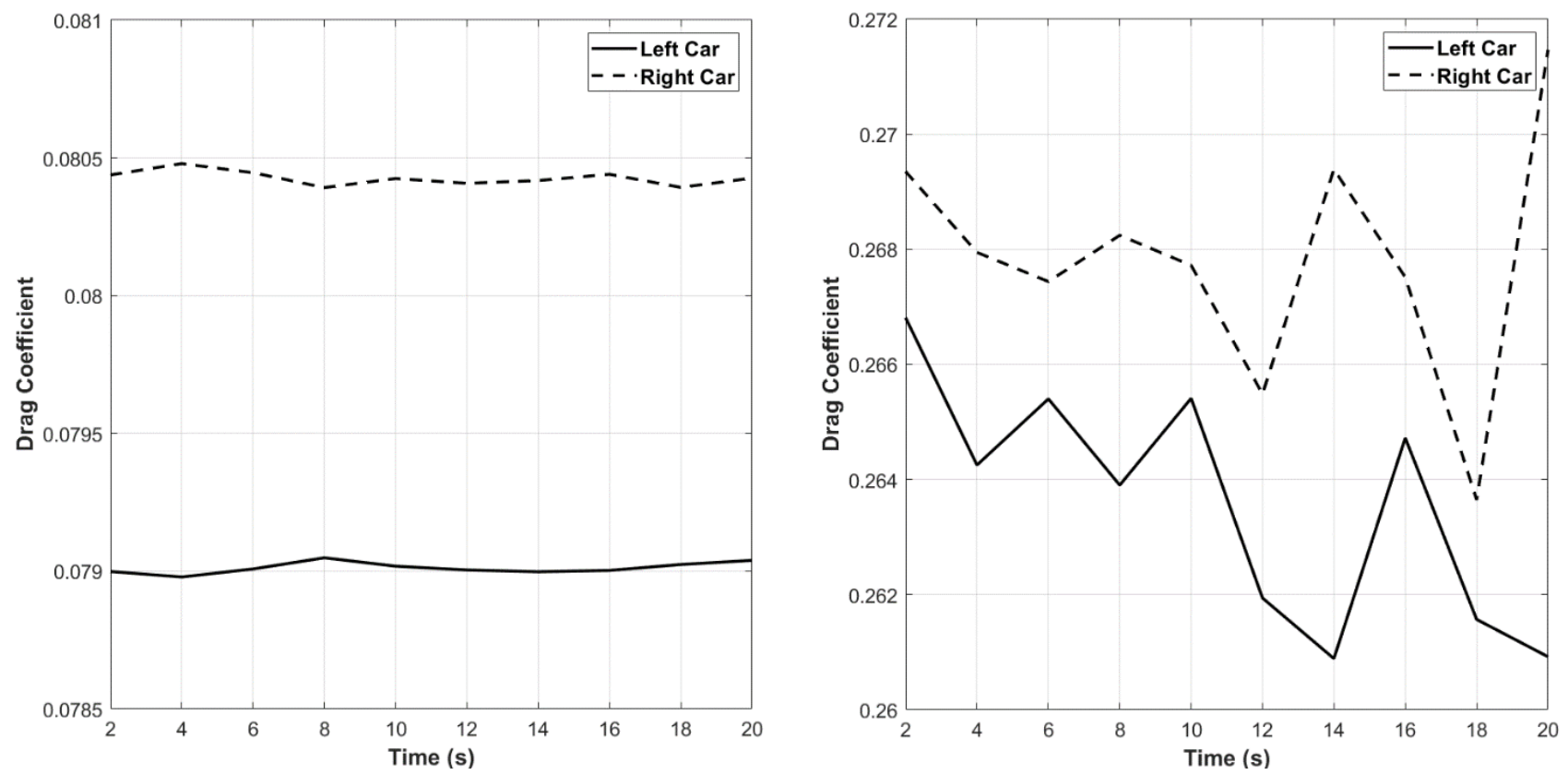

Fig. 9: Drag coefficient of cars in transient mode: a) $0.2 \mathrm{~m}$ lateral distance b) $1 \mathrm{~m}$ lateral distance
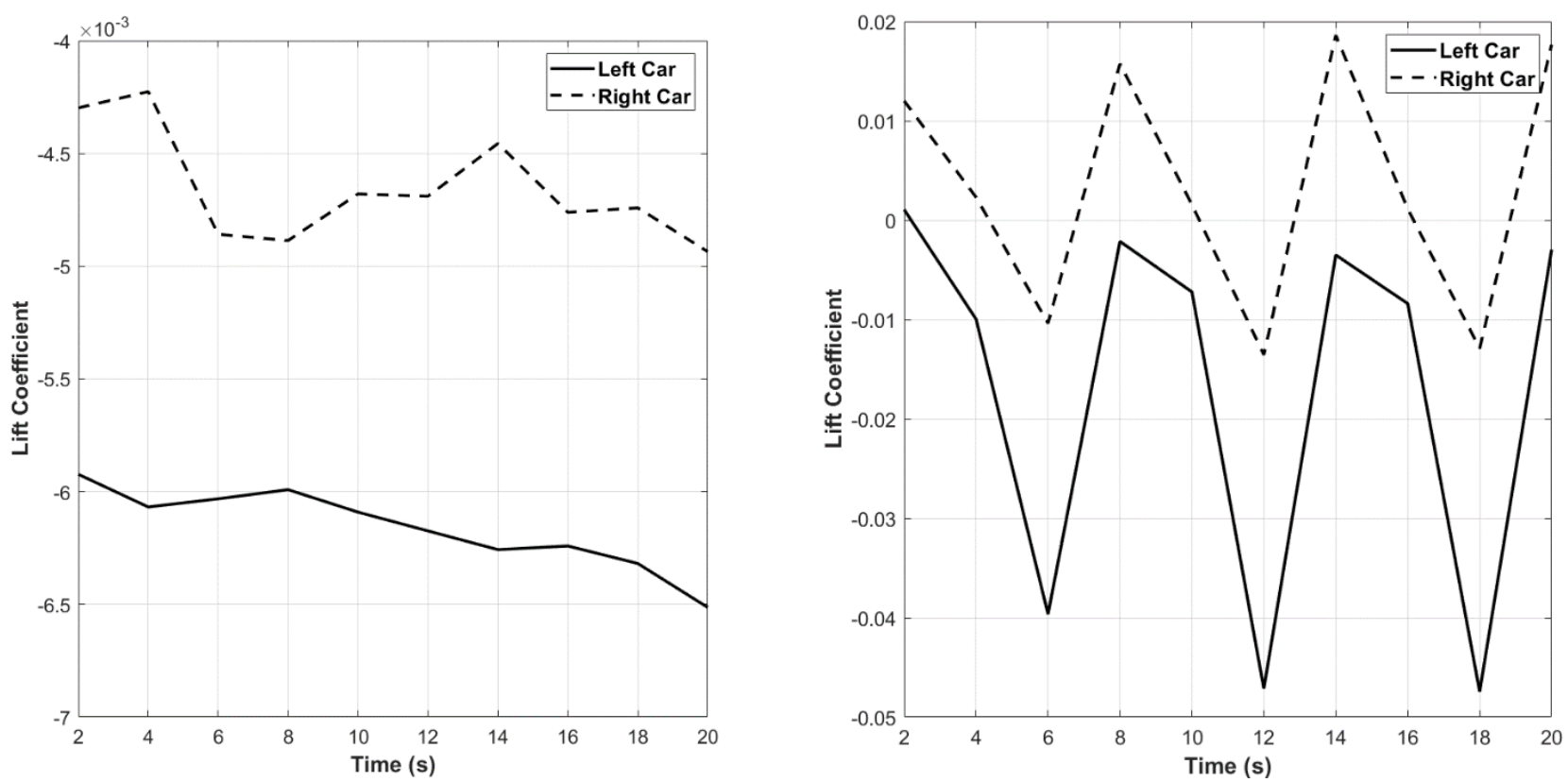

Fig. 10: Lift coefficient of cars in transient mode: a) $0.2 \mathrm{~m}$ lateral distance b) $1 \mathrm{~m}$ lateral distance 
To investigate the influence of cars on each other, pressure and velocity contour on the plane of $0.2 \mathrm{~m}$ above the floor is shown in the following figures. By comparing pressure contour, it can be concluded that the low-pressure region is more significant in the rear of cars in the fewer lateral distances, so they have a greater drag coefficient. By comparing the velocity contours, it can be concluded that the fluid flow behind cars is more symmetrical in the farther distance.

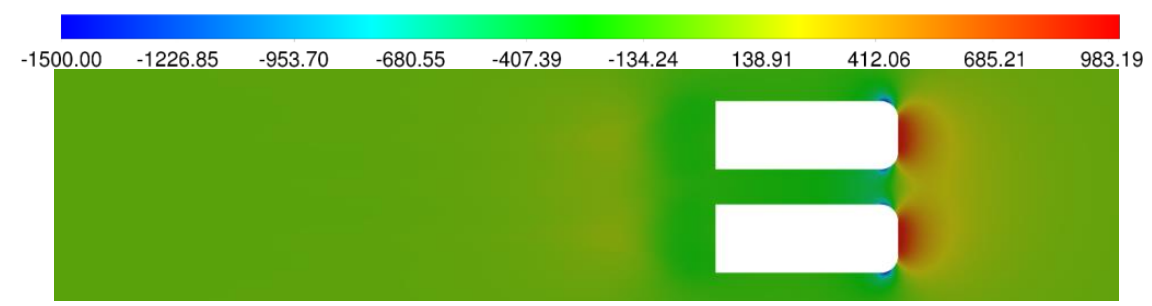

Fig. 11: Static pressure contour of $0.2 \mathrm{~m}$ lateral distance

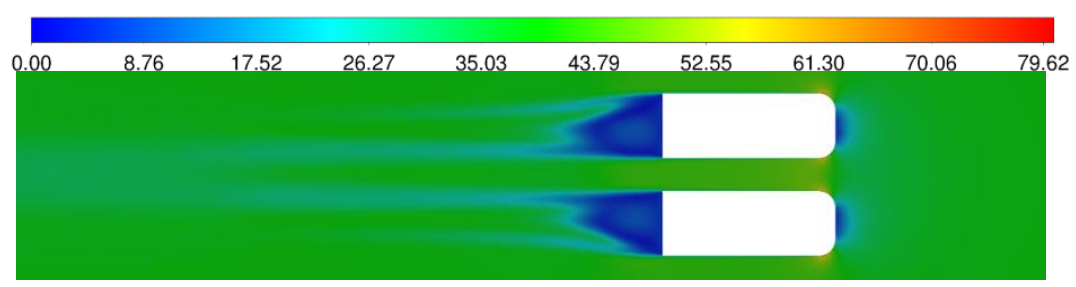

Fig. 12: Velocity contour of $0.2 \mathrm{~m}$ lateral distance

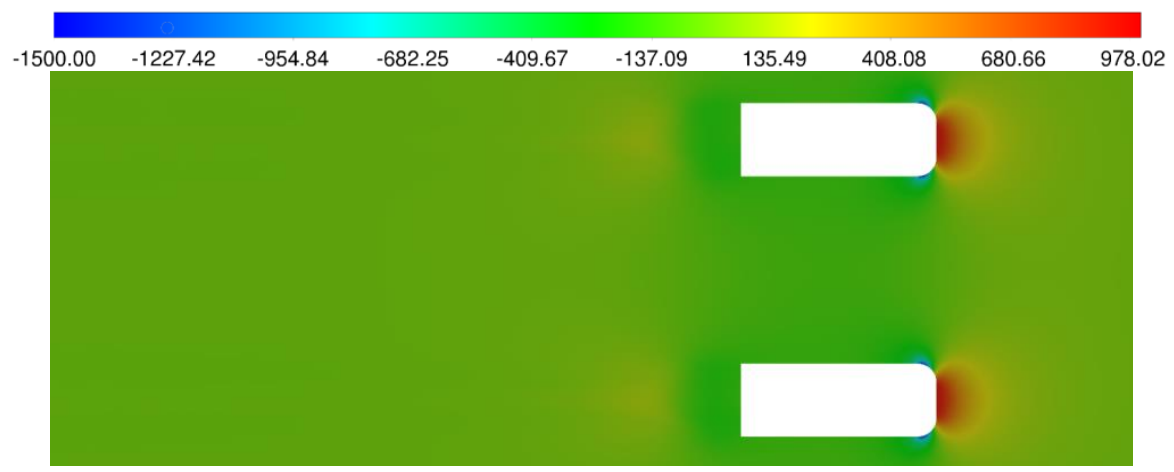

Fig. 13: Static pressure contour of $1 \mathrm{~m}$ lateral distance

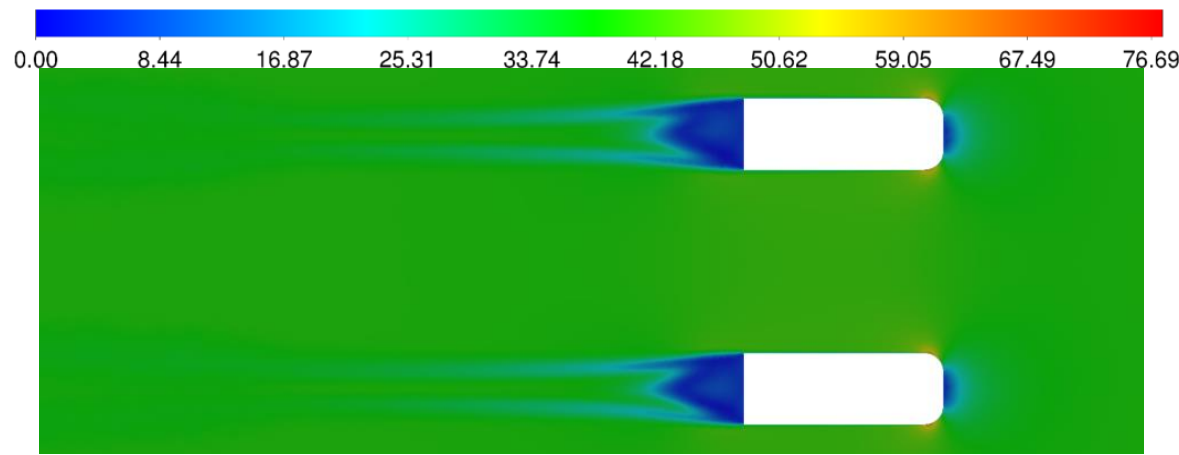

Fig. 14: Velocity contour of $1 \mathrm{~m}$ lateral distance 


\section{Conclusion}

To investigate the influence of one car on the aerodynamic coefficients of another car, which moves closely, the present study chose the Ahmed body as the benchmark vehicle to pursue the present objectives. Suitable grids were produced using the tetrahedron method. The realizable $k$-epsilon turbulence model with enhanced wall function for the near-wall treatment was considered in this study. In the longitudinal distance study, it was observed that the drag coefficient of both cars would significantly decrease for the front car for the short distance case. Also, the lift coefficient of both cars reduced, and this value even was negative for the rear car. In the investigation of lateral distance, the drag coefficient depended on the actual distance. In shorter distance case, this value showed increase and in long-distance, it showed decrease. The lift coefficient had reduction in both short and long-distances. In the lateral distance case, it was expected to arrive at equal lift and drag coefficients for both left and right cars; however, in practice, there was slight difference between them, which could be attributed to asymmetry in the generated unstructured mesh.

\section{Acknowledgment}

We gratefully acknowledge Mr. Pooya Javadpour, who assisted us in accomplishing this paper. The authors would like to acknowledge the financial grant received from the Department of Research and Technology of Sharif University of Technology.

\section{References}

[1] Ahmed, "Some Salient Features of the Time - Averaged Ground Vehicle Wake," Society of Automotive Engineers, 1984.

[2] W. Meile, G. Brenn, A. Reppenhagen and B. Lechner, "Experiments and numerical simulations on the aerodynamics of the Ahmed body," CFD Letters, 2011.

[3] V. Naveen Kumar, Dr. K. Lalit Narayan, L. N. V. Narasimha Rao and Y. Sri Ram, "Investigation of Drag and Lift Forces over the Profile of Car with Rearspoiler Using CFD," International Journal of Science and Research, 2013.

[4] B. Blocken and Y. Toparlar, "A following car influences cyclist drag: CFD simulations and wind tunnel measurements," Wind Engineering and Industrial Aerodynamics, 2015.

[5] M. Minguez, R. Pasquetti and E. Serre, "High-order large-eddy simulation of flow over the "Ahmed body" car model," Physics of Fluids, 2008.

[6] S. Banga, Md. Zunaid, N. Ahmad Ansari, S. Sharma and R. S. Dungriya, "CFD Simulation of Flow around External Vehicle: Ahmed Body," Journal of Mechanical and Civil Engineering, 2015.

[7] B. Mohammadikalakoo, P. Schito and M. Mani, "Passive flow control on Ahmed body by rear linking tunnels," Journal of Wind Engineering and Industrial Aerodynamics, 2020.

[8] N. A. Siddiqui and M. Agelin-Chaab, "Effect of aspect ratio on the recirculation region of $35^{\circ}$ Ahmed body," Australian Journal of Mechanical Engineering, 2020.

[9] Filipe F.Buscarioloa, Spencer J.Sherwin and Gustavo R.S.Assi, "Computational study on an Ahmed Body equipped with simplified underbody diffuser," Journal of Wind Engineering and Industrial Aerodynamics, 2021.

[10] H. Pletcher, C. Tannehill and A. Anderson, Computational Fluid Mechanics and Heat Transfer, CRS Press, 2013.

[11] M. Rouméas, P. Gilliéron and A. Kourta, "Drag Reduction by Flow Separation Control on a Car After Body," Numer. Methods Fluids, 2008 